\title{
The Odd Lindley-G Family of Distributions
}

\author{
Frank Gomes-Silva \\ Federal R. Univ. of Pernambuco \\ Manoel W. Ramos \\ Federal Institute of Paraíba
}

\author{
Ana Percontini \\ State Univ. of F. de Santana \\ Ronaldo Venâncio \\ Recife Military School
}

\author{
Edleide de Brito \\ Federal Univ. of Bahia \\ Gauss Cordeiro \\ Federal Univ. of Pernambuco
}

\begin{abstract}
We propose a new generator of continuous distributions with one extra positive parameter called the odd Lindley-G family. Some special cases are presented. The new density function can be expressed as a linear combination of exponentiated densities based on the same baseline distribution. Various structural properties of the new family, which hold for any baseline model, are derived including explicit expressions for the quantile function, ordinary and incomplete moments, generating function, Rényi entropy, reliability, order statistics and their moments and $k$ upper record values. We provide a Monte Carlo simulation study to evaluate the maximum likelihood estimates. We discuss estimation of the model parameters by maximum likelihood and provide an application to a real data set.
\end{abstract}

Keywords: estimation, Lindley distribution, generating function, odd Lindley-G distribution; $k$ upper record values, moment, Monte Carlo simulation.

\section{Introduction}

In recent years, several ways of generating new distributions from classic ones has attracted theoretical and applied statisticians due to their flexible properties. Many classical distributions have been extensively used over the past decades for modeling data in several areas. In fact, for instance, Johnson, Kotz, and Balakrishnan $(1994,1995)$ presented a comprehensive discussion on hundreds of continuous univariate distributions. However, in many applied areas including, but not limited to lifetime analysis, finance and insurance, there is a clear need for extended forms of these distributions, which are more flexible for fitting specific real world scenarios. Consequently, recent developments focus on definition of the new families of distributions that extend well-known distributions and at the same time provide great flexibility in modelling data in practice. Some well-established generators and other recently proposed are the Marshall-Olkin generated family (MO-G) by Marshall and Olkin (1997), beta-G by Eugene, Lee, and Famoye (2002), Kumaraswamy-G (Kw-G for short) by Cordeiro and de Castro (2011), McDonald-G (Mc-G) by Alexander, Cordeiro, Ortega, and Sarabia (2012), gamma-G by Zografos and Balakrishnan (2009), transformed-transformer (T-X) by Alzaatreh, Lee, and Famoye (2013), exponentiated T-X by Alzaghal, Felix, and Carl (2013), Weibull-G by Bourguignon, Silva, and Cordeiro (2014), exponentiated half-logistic family by Cordeiro, Alizadeh, and Ortega (2014), logistic-X by Tahir, Cordeiro, Alzaatreh, Mansoor, 
and Zubair (2016a), a new Weibull-G by Tahir, Zubair, Mansoor, Cordeiro, Alizadeh, and Hamedani (2016b) and Kumaraswamy odd log-logistic-G by Alizadeh, Emadi, Doostparast, Cordeiro, Ortega, and Pescim (2015). The Lindley distribution was originally proposed by Lindley (1958) as a counterexample of fiducial statistics. Ghitany, Atieh, and Nadarajah (2008) showed through a numerical example that the hazard function of the Lindley distribution does not exhibit a constant hazard rate, indicating its flexibility over the exponential distribution. It has recently received considerable attention as an appropriate model to analyze lifetime data especially in applications modeling stress-strength reliability; see, for example, Ghitany et al. (2008), Zakerzadeh and Dolati (2009), Mazucheli and Achcar (2011), Gupta and Singh (2012), Warahena-Liyanage and Pararai (2014). Several other authors including Sankaran (1970), Nadarajah and Tahmasbi (2011) and Asgharzedah, Bakouch, and Esmaeli (2013) developed some structural properties of various generalized Lindley distributions. Nonetheless, there are situations in which the Lindley distribution and all of its generalizations may not be suitable from a theoretical or an applied point of view.

Let $r(t)$ be the probability density function (pdf) of a random variable $T \in[a, b]$ for $-\infty \leq$ $a<b<\infty$ and let $W[G(x)]$ be a function of the cumulative distribution function (cdf) of a random variable $X$ such that $W[G(x)]$ satisfies the following conditions:

$$
\begin{cases}(i) & W[G(x)] \in[a, b], \\ (\text { ii }) & W[G(x)] \text { is differentiable and monotonically non-decreasing, and } \\ (\text { iii }) & W[G(x)] \rightarrow a \text { as } x \rightarrow-\infty \text { and } W[G(x)] \rightarrow b \text { as } x \rightarrow \infty .\end{cases}
$$

Alzaatreh et al. (2013) defined the T-X family of distributions by

$$
F(x)=\int_{a}^{W[G(x)]} r(t) d t
$$

where $W[G(x)]$ satisfies the conditions (1). The pdf corresponding to (2) is given by

$$
f(x)=\left\{\frac{d}{d x} W[G(x)]\right\} r\{W[G(x)]\} .
$$

In this paper, we propose a new wider class of continuous distributions called the Odd Lindley$G$ ("OL-G" for short) family by taking $W[G(x)]=\frac{G(x ; \boldsymbol{\xi})}{1-G(x ; \boldsymbol{\xi})}$ and $r(t)=\frac{a^{2}}{1+a}(1+t) \mathrm{e}^{-a t}, t>$ $0, a>0$, where $G(x ; \boldsymbol{\xi})$ is a baseline cdf, which depends on a parameter vector $\boldsymbol{\xi}$ and $\bar{G}(x ; \boldsymbol{\xi})=$ $1-G(x ; \boldsymbol{\xi})$ is the baseline survival function. Its cdf is given by

$$
\begin{aligned}
F(x ; a, \boldsymbol{\xi}) & =\int_{0}^{\frac{G(x ; \boldsymbol{\xi})}{1-G(x ; \boldsymbol{\xi})}} \frac{a^{2}}{1+a}(1+t) \mathrm{e}^{-a t} d t \\
& =1-\frac{a+\bar{G}(x ; \boldsymbol{\xi})}{(1+a) \bar{G}(x ; \boldsymbol{\xi})} \exp \left\{-a \frac{G(x, \boldsymbol{\xi})}{\bar{G}(x ; \boldsymbol{\xi})}\right\} .
\end{aligned}
$$

For each baseline G, the OL-G family of distributions is defined by the cdf (4). Equation (4) is a wider family of continuous distributions. Further, we can omit sometimes the dependence on the vector $\boldsymbol{\xi}$ of the parameters and write simply $G(x)=G(x ; \boldsymbol{\xi})$.

The corresponding density function to (4) is given by

$$
f(x ; a, \boldsymbol{\xi})=\frac{a^{2}}{(1+a)} \frac{g(x, \boldsymbol{\xi})}{\bar{G}(x ; \boldsymbol{\xi})^{3}} \exp \left\{-a \frac{G(x, \boldsymbol{\xi})}{\bar{G}(x ; \boldsymbol{\xi})}\right\},
$$

where $g(x ; \boldsymbol{\xi})$ is the baseline pdf. Equation (5) is most tractable when the $\operatorname{cdf} G(x)$ and the pdf $g(x)$ have simple analytic expressions. Hereafter, a random variable $X$ with density function (5) is denoted by $X \sim \operatorname{OL}-\mathrm{G}(a, \boldsymbol{\xi})$. Table 1 lists $G(x ; \boldsymbol{\xi}) / \bar{G}(x ; \boldsymbol{\xi})$ and the corresponding parameters for some special distributions. 
Most of the distributions lack physical motivation for modeling lifetime data. We now provide, in a similar context, a physical interpretation for the proposed family inspired in Cooray (2006). Let $Y$ be a lifetime random variable having a certain continuous $\mathrm{G}$ distribution. The odds ratio that an individual (or component) following the lifetime $Y$ will die (failure) at time $x$ is $G(x ; \boldsymbol{\xi}) / \bar{G}(x ; \boldsymbol{\xi})$. Consider that the variability of this odds of death is represented by the random variable $X$ and assume that it follows the Lindley model with scale $a$. We can write

$$
\operatorname{Pr}(Y \leq x)=\operatorname{Pr}\left(X \leq \frac{G(x ; \boldsymbol{\xi})}{\bar{G}(x ; \boldsymbol{\xi})}\right)=F(x ; a, \boldsymbol{\xi}),
$$

which is given by (4). The rest of the paper is organized as follows. In Section 2, we present

Table 1: Distributions and corresponding $G(x ; \boldsymbol{\xi}) / \bar{G}(x ; \boldsymbol{\xi})$ functions

\begin{tabular}{lll}
\hline Distribution & $G(x ; \boldsymbol{\xi}) / \bar{G}(x ; \boldsymbol{\xi})$ & $\boldsymbol{\xi}$ \\
\hline Uniform $(0<x<\theta)$ & $x /(\theta-x)$ & $\theta$ \\
Exponential $(x>0)$ & $\mathrm{e}^{\lambda x}-1$ & $\lambda$ \\
Weibull $(x>0)$ & $\mathrm{e}^{\lambda x^{\gamma}-1}$ & $(\lambda, \gamma)$ \\
Fréchet $(x>0)$ & $\left(\mathrm{e}^{\left.\lambda x^{\gamma}-1\right)^{-1}}\right.$ & $(\lambda, \gamma)$ \\
Half-logistic $(x>0)$ & $\left(\mathrm{e}^{x}-1\right) / 2$ & $\varnothing$ \\
Power function $(0<x<1 / \theta)$ & {$\left[(\theta x)^{-k}-1\right]^{-1}$} & $(\theta, k)$ \\
Pareto $(x \geq \theta)$ & $(x / \theta)^{k}-1$ & $(\theta, k)$ \\
Burr XII $(x>0)$ & {$\left[1+(x / s)^{c}\right]^{k}-1$} & $(s, k, c)$ \\
Log-logistic $(x>0)$ & {$\left[1+(x / s)^{c}\right]-1$} & $(s, c)$ \\
Lomax $(x>0)$ & {$[1+(x / s)]^{k}-1$} & $(s, k)$ \\
Gumbel $(-\infty<x<\infty)$ & $\{\exp [\exp (-(x-\mu) / \sigma)]-1\}^{-1}$ & $(\mu, \sigma)$ \\
Kumaraswamy $(0<x<1)$ & $\left(1-x^{\alpha}\right)^{-\beta}-1$ & $(\alpha, \beta)$ \\
Normal $(-\infty<x<\infty)$ & $\Phi((x-\mu) / \sigma) /(1-\Phi((x-\mu) / \sigma))$ & $(\mu, \sigma)$ \\
\hline
\end{tabular}

four special models of the new family. A range of mathematical properties of Equation (5) is derived in Section 3 including a useful expansion for the pdf and explicit expressions for the moments and generating function. General expressions for the Rényi entropy, reliability, order statistics and $k$ upper record values are discussed in Section 4. Estimation of the model parameters by maximum likelihood is performed in Section 5. In Section 6, we conduct a simulation study for specific choices of a model parameter. An application to a real data set illustrates the performance of the new family in Section 6. The paper is concluded in Section 7.

\section{Four special models of the OL-G family}

In this section, we present four special models of the OL-G family.

\subsection{Odd Lindley Weibull (OLW)}

The Weibull cdf with parameters $\alpha>0$ and $\lambda>0$ is $G(x)=1-\mathrm{e}^{-(\lambda x)^{\alpha}}$ (for $x>0$ ). The cdf of a random variable $X$ having the OLW distribution, say $X \sim \operatorname{OLW}(a, \alpha, \lambda)$, is given by

$F_{\text {OLW }}(x)=(1+a)^{-1} \exp \left\{-a\left[a+\mathrm{e}^{(\lambda x)^{\alpha}}\right]\right\}\left\{\left(1+a+a^{2}\right) \exp \left[a \mathrm{e}^{(\lambda x)^{\alpha}}\right]-\mathrm{e}^{a(1+a)}\left[1+a \mathrm{e}^{(\lambda x)^{\alpha}}\right]\right\}$,

and the associated density function reduces to

$$
f_{\mathrm{OLW}}(x)=a^{2}(1+a)^{-1} \alpha \lambda^{\alpha} x^{\alpha-1} \mathrm{e}^{2(\lambda x)^{\alpha}} \exp \left\{-a\left[\mathrm{e}^{(\lambda x)^{\alpha}}-1\right]\right\} .
$$


The hazard rate function (hrf) corresponding to (6) is given by

$$
\begin{aligned}
\tau_{\mathrm{OLW}}(x) & =a^{2} \alpha \lambda^{\alpha} x^{\alpha-1} \mathrm{e}^{2(\lambda x)^{\alpha}} \exp \left\{-a\left[\mathrm{e}^{(\lambda x)^{\alpha}}-1\right]\right\}\left\{1+a-\exp \left\{-a\left[a+\mathrm{e}^{(\lambda x)^{\alpha}}\right]\right\}\right. \\
& \left.\times\left[\left(1+a+a^{2}\right) \exp \left[a \mathrm{e}^{(\lambda x)^{\alpha}}\right]-\mathrm{e}^{a(1+a)}\left\{1+a \mathrm{e}^{(\lambda x)^{\alpha}}\right\}\right]\right\}^{-1} .
\end{aligned}
$$

Plots of the density and hrf of the OLW distribution for some parameter values are displayed in Figure 1.
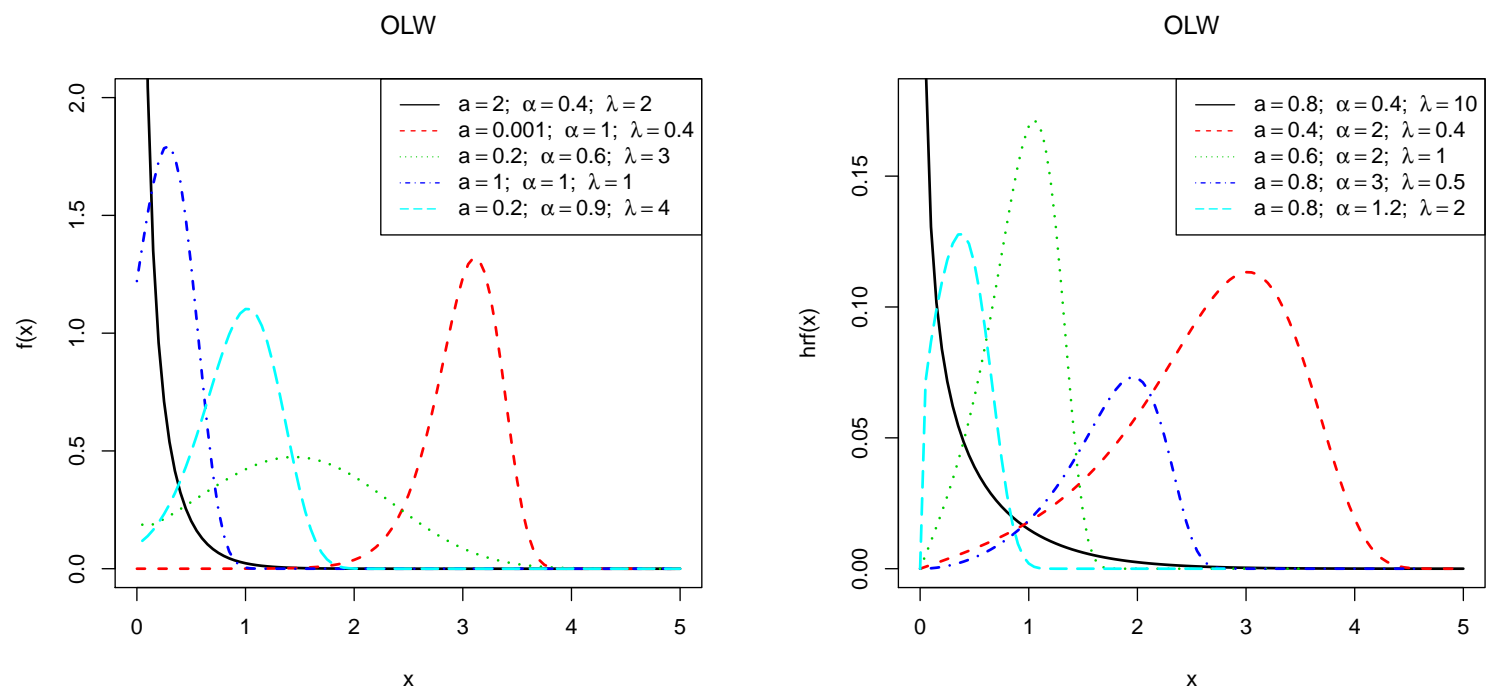

Figure 1: Plots of the OLW density and hrf functions for some parameter values.

\subsection{Odd Lindley Kumaraswamy (OLKw)}

The Kumaraswamy cumulative distribution (for $x \in[0,1]$ ) is $G(x)=1-\left(1-x^{\alpha}\right)^{\beta}$, where the parameters are $\alpha>0$ and $\beta>0$. The OLKw cumulative distribution is given by

$$
F_{\text {OLKw }}(x)=(1+a)^{-1}\left\{\left(1+a+a^{2}\right) \mathrm{e}^{-a^{2}}+\exp \left[a-a\left(1-x^{\alpha}\right)^{-\beta}\right]\left[-1-a\left(1-x^{\alpha}\right)^{-\beta}\right]\right\}
$$

and the associated density function reduces to

$$
f_{\mathrm{OLKw}}(x)=a^{2}(1+a)^{-1} \alpha \beta x^{\alpha-1}\left(1-x^{\alpha}\right)^{-2 \beta-1} \exp \left\{-a\left[\left(1-x^{\alpha}\right)^{-\beta}-1\right]\right\} .
$$

The hrf corresponding to (7) is given by

$$
\begin{aligned}
\tau_{\mathrm{OLKW}}(x) & =\alpha \beta x^{\alpha-1}\left(1-x^{\alpha}\right)^{-2 \beta-1} \exp \left\{-a\left[\left(1-x^{\alpha}\right)^{-\beta}-1\right]\right\} \\
& \times\left\{1+a-\left[\left(1+a+a^{2}\right) \mathrm{e}^{-a^{2}}+\exp \left[a-a\left(1-x^{\alpha}\right)^{-\beta}\right]\left\{-1-a\left(1-x^{\alpha}\right)^{-\beta}\right\}\right]\right\}^{-1} .
\end{aligned}
$$

Plots of the density and hrf of the OLKw distribution for some parameter values are displayed in Figure 2.

\subsection{Odd Lindley half-logistic (OLHL)}

The half-logistic cumulative distribution (for $x>0$ ) is given by $G(x)=\frac{1-\mathrm{e}^{-x}}{1+\mathrm{e}^{-x}}$. The OLHL cumulative distribution becomes

$$
\begin{aligned}
F_{\text {OLHL }}(x) & =[2(1+a)]^{-1} \exp \left[\frac{a}{2}\left(1-2 a-\mathrm{e}^{x}\right)\right] \\
& \times\left\{-(2+a) \mathrm{e}^{a^{2}}+2\left(1+a+a^{2}\right) \exp \left[\frac{a}{2}\left(-1+\mathrm{e}^{x}\right)\right]-a \mathrm{e}^{a^{2}+x}\right\}
\end{aligned}
$$



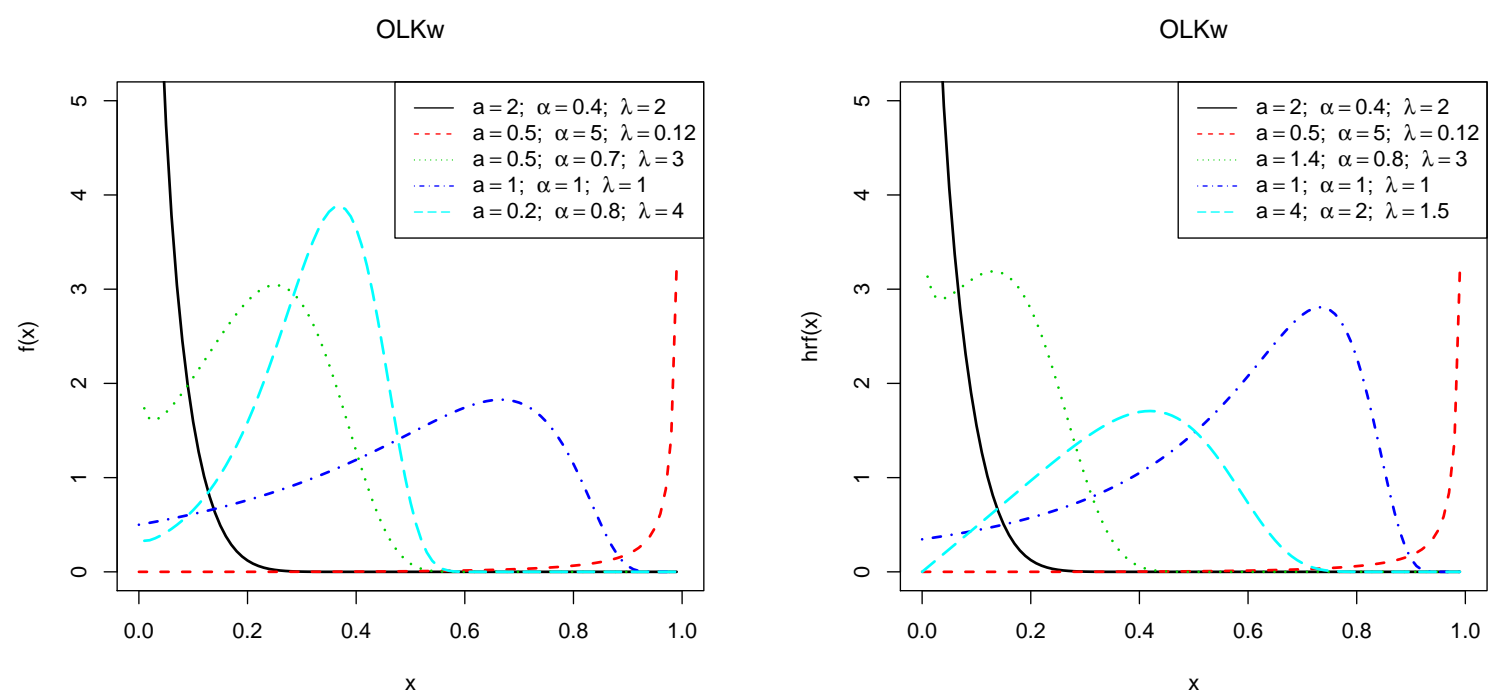

Figure 2: Plots of the OLKw density and hrf functions for some parameter values.

and the associated density function reduces to

$$
f_{\mathrm{OLHL}}(x)=a^{2}[4(1+a)]^{-1}\left(1+\mathrm{e}^{x}\right) \exp \left[\frac{a}{2}\left(1-\mathrm{e}^{x}\right)+x\right] .
$$

The corresponding hrf to (8) is given by

$$
\begin{aligned}
\tau_{\mathrm{OLHL}}(x) & =\frac{a^{2}}{2}\left(1+\mathrm{e}^{x}\right) \exp \left[\frac{a}{2}\left(1-\mathrm{e}^{x}\right)+x\right]\left\{2(1+a)-\exp \left[\frac{a}{2}\left(1-2 a-\mathrm{e}^{x}\right)\right]\right. \\
& \left.\times\left[-(2+a) \mathrm{e}^{a^{2}}+2\left(1+a+a^{2}\right) \exp \left[\frac{a}{2}\left(-1+\mathrm{e}^{x}\right)\right]-a \mathrm{e}^{a^{2}+x}\right]\right\}^{-1} .
\end{aligned}
$$

Plots of the pdf and hrf of the OLHL distribution for some parameter values are displayed in Figure 3 .

OLHL

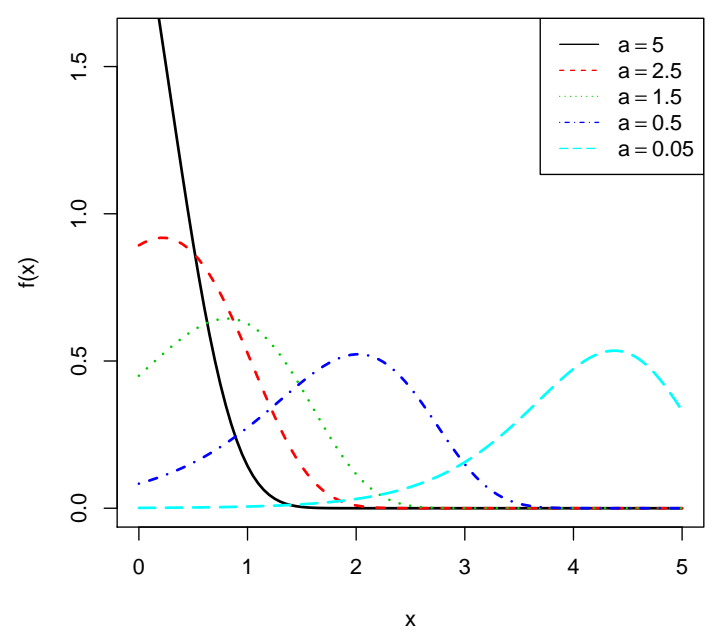

OLHL

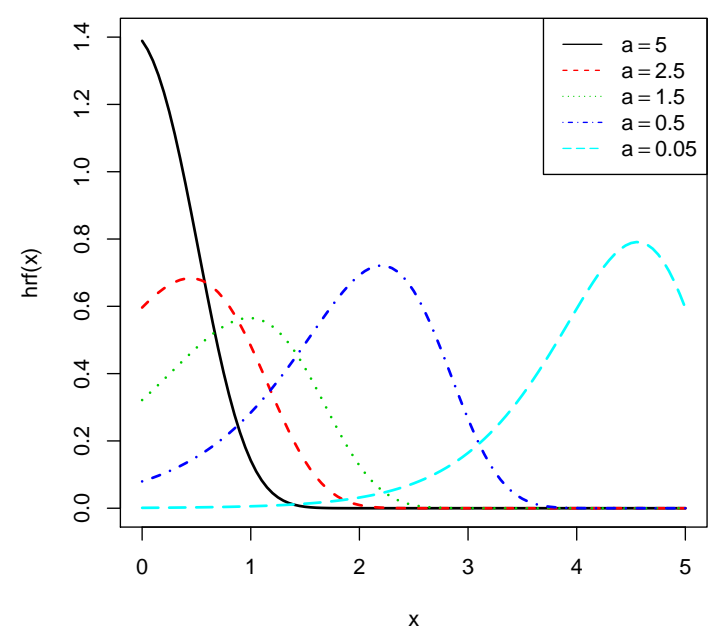

Figure 3: Plots of the OLHL density and hrf functions for some parameter values. 


\subsection{Odd Lindley Burr XII (OLBXII)}

Zimmer, Keats, and Wang (1998) introduced the three parameter Burr XII (BXII) distribution with cdf and pdf (for $x>0$ ): $G(x ; s, k, c)=1-\left[1+\left(\frac{x}{s}\right)^{c}\right]^{-k}$ and $g(x ; s, k, c)=$ $c k s^{-c} x^{c-1}\left[1+\left(\frac{x}{s}\right)^{c}\right]^{-k-1}$, respectively, where $k>0$ and $c>0$ are shape parameters and $s>0$ is a scale parameter. The OLBXII cumulative distribution becomes

$$
\begin{aligned}
F_{\text {OLBXII }}(x) & =(1+a)^{-1} \exp \left\{a+\left[1+\left(\frac{x}{s}\right)^{c}\right]^{k}\right\} \\
& \times\left\{\left(1+a+a^{2}\right) \exp \left\{a\left[1+\left(\frac{x}{s}\right)^{c}\right]^{k}\right\}-\mathrm{e}^{a(1+a)}\left(1+a\left[1+\left(\frac{x}{s}\right)^{c}\right]^{k}\right)\right\}
\end{aligned}
$$

and the associated density function reduces to

$$
f_{\text {OLBXII }}(x)=a^{2} c k s^{-c}(1+a)^{-1} x^{c-1}\left[1+\left(\frac{x}{s}\right)^{c}\right]^{2 k-1} \exp \left[a\left\{1-\left[1+\left(\frac{x}{s}\right)^{c}\right]^{k}\right\}\right] .
$$

The corresponding hrf is given by

$$
\begin{aligned}
\tau_{\text {OLBXII }}(x) & =a^{2} c k s^{-c} x^{c-1}\left[1+\left(\frac{x}{s}\right)^{c}\right]^{2 k-1} \exp \left[a\left\{1-\left[1+\left(\frac{x}{s}\right)^{c}\right]^{k}\right\}\right] \\
& \times\left\{1+a-\exp \left\{a+\left[1+\left(\frac{x}{s}\right)^{c}\right]^{k}\right\}\right. \\
& \left.\times\left[\left(1+a+a^{2}\right) \exp \left\{a\left[1+\left(\frac{x}{s}\right)^{c}\right]^{k}\right\}-\mathrm{e}^{a(1+a)}\left(1+a\left[1+\left(\frac{x}{s}\right)^{c}\right]^{k}\right)\right]\right\}^{-1} .
\end{aligned}
$$

Plots of the density and hrf of the OLBXII distribution for some parameter values are displayed in Figure 4.
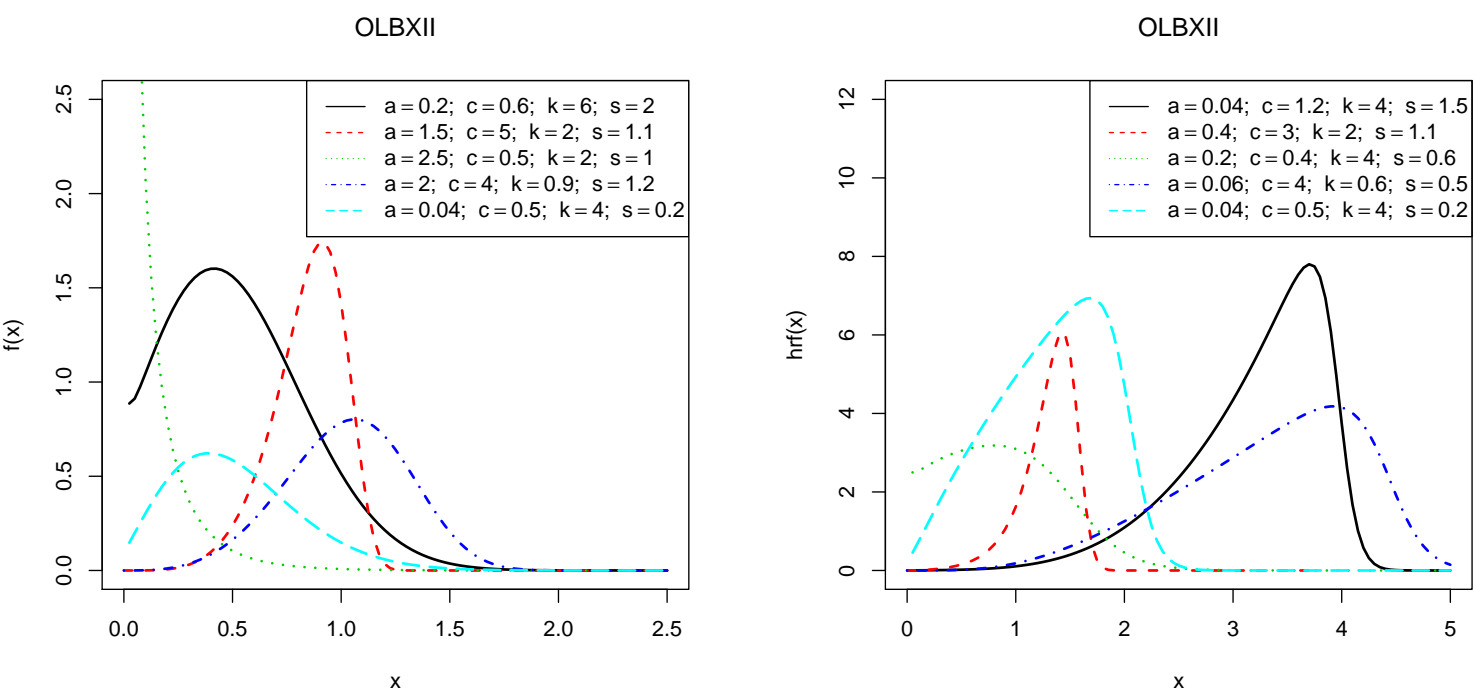

Figure 4: Plots of the OLBXII density and hrf functions for some parameter values.

\section{Main properties}

\subsection{Survival and hazard}

The corresponding survival function to (4) is given by

$$
S(x ; a, \boldsymbol{\xi})=1-F(x ; a, \boldsymbol{\xi})=\frac{a+\bar{G}(x ; \boldsymbol{\xi})}{(1+a) \bar{G}(x ; \boldsymbol{\xi})} \exp \left\{-a \frac{G(x, \boldsymbol{\xi})}{\bar{G}(x ; \boldsymbol{\xi})}\right\} .
$$


The hrf of $X$ becomes

$$
\tau(x ; a, \boldsymbol{\xi})=\frac{a^{2} g(x, \boldsymbol{\xi})}{\bar{G}(x ; \boldsymbol{\xi})^{2}[a+\bar{G}(x ; \boldsymbol{\xi})]}=\frac{a^{2}}{\bar{G}(x ; \boldsymbol{\xi})[a+\bar{G}(x ; \boldsymbol{\xi})]} \tau(x ; \boldsymbol{\xi}),
$$

where $\tau(x ; \boldsymbol{\xi})=g(x ; \boldsymbol{\xi}) / \bar{G}(x ; \boldsymbol{\xi})$. The multiplying quantity $a^{2} /\{\bar{G}(x ; \boldsymbol{\xi})[a+\bar{G}(x ; \boldsymbol{\xi})]\}$ works as a corrected factor for the baseline hrf. Equation (4) can deal with general situations in modeling survival data with various shapes of the hrf.

\subsection{Quantile functions}

Let $X$ be an arbitrary random variable with cdf $F(x)=\operatorname{Pr}(X \leq x)$, where $x \in \mathbb{R}$. For any $u \in(0,1)$, the $u$ th quantile function (qf) $Q(u)$ of $X$ is the solution of

$$
F(Q(u))=u,
$$

for $Q(u)>0$.

For any fixed $a>0$, from Equation (4), we obtain

$$
-\frac{1+a-G(Q(u))}{1-G(Q(u))} \mathrm{e}^{-\frac{a G(Q(u))}{1-G(Q(u))}}=(1+a)(u-1)
$$

Multiplying both sides of this equation by $\mathrm{e}^{-(1+a)}$ gives

$$
-\frac{1+a-G(Q(u))}{1-G(Q(u))} \mathrm{e}^{-\frac{1+a-G(Q(u))}{1-G(Q(u))}}=(1+a)(u-1) \mathrm{e}^{-(1+a)} .
$$

In the above equation, we note that $-\frac{1+a-G(Q(u))}{1-G(Q(u))}$ is the Lambert $\mathrm{W}$ function of the real argument $(1+a)(u-1) \mathrm{e}^{-(1+a)}$. The Lambert $\mathrm{W}$ function is defined by

$$
W(x) \mathrm{e}^{W(x)}=x
$$

The Lambert function has two real branches with a branching point located at $\left(-\mathrm{e}^{-1}, 1\right)$. The lower branch, $W_{-1}(x)$, is defined in the interval $\left[-\mathrm{e}^{-1}, 1\right]$ and has a negative singularity for $x \rightarrow 0^{-}$. The upper branch, $W_{0}(x)$, is defined for $x \in\left[-\mathrm{e}^{-1}, \infty\right]$.

Then, we have

$$
W\left((1+a)(u-1) \mathrm{e}^{-(1+a)}\right)=-\left(1+\frac{a}{1-G(Q(u))}\right)
$$

Clearly, for any $a>0$ and $u \in(0,1)$, we have $\left(1+\frac{a}{1-G(Q(u))}\right)>1$ and then $(1+a)(u-$ $1) \mathrm{e}^{-(1+a)}<0$. Therefore, considering the lower branch of the Lambert $\mathrm{W}$ function, we can write (13) as

$$
W_{-1}\left((1+a)(u-1) \mathrm{e}^{-(1+a)}\right)=-\left(1+\frac{a}{1-G(Q(u))}\right) .
$$

Hence, the qf of $X$ is given by

$$
Q(u)=G^{-1}\left\{1+a\left[1+W_{-1}\left((1+a)(u-1) \mathrm{e}^{-(1+a)}\right)\right]^{-1}\right\} .
$$




\subsection{Shapes of the OL-G family}

The shapes of the density and hazard rate functions can also be described analytically. The critical points of the OL-G density function are the roots of the equation:

$$
\frac{g^{\prime}(x)}{g(x)}+3 \frac{g(x)}{\bar{G}(x)}-a \frac{g(x)}{\bar{G}(x)^{2}}=0 .
$$

The critical points of $h(x)$ are obtained from the following equation

$$
\frac{g^{\prime}(x)}{g(x)}+\frac{g(x)}{a+\bar{G}(x)}+2 \frac{g(x)}{\bar{G}(x)^{2}}=0 .
$$

By using most computer algebra systems, we can examine Equations (15) and (16) to determine the local maximums and minimums and inflexion points.

\subsection{Useful expansions}

Several structural properties of the extended distributions may be easily explored using mixture forms of exponentiated-G ("Exp-G") distributions. In this section, we obtain expansions for $f(x)$ and $F(x)$. First, we define the Exp-G distribution for an arbitrary parent distribution $G(x)$, say $W \sim \operatorname{Exp}-\mathrm{G}(c)$, if $W$ has cdf and pdf given by

$$
H_{c}(x ; \boldsymbol{\xi})=G(x ; \boldsymbol{\xi})^{c} \quad \text { and } \quad h_{c}(x ; \boldsymbol{\xi})=c g(x ; \boldsymbol{\xi}) G(x ; \boldsymbol{\xi})^{c-1},
$$

respectively. Next, we obtain an expansion for $f(x)$. Using the power series for the exponential function, we have

$$
\exp \left\{-a\left[\frac{G(x ; \boldsymbol{\xi})}{\bar{G}(x ; \boldsymbol{\xi})}\right]\right\}=\sum_{k=0}^{\infty} \frac{(-1)^{k} a^{k}}{k !}\left[\frac{G(x ; \boldsymbol{\xi})}{\bar{G}(x ; \boldsymbol{\xi})}\right]^{k} .
$$

Inserting this expansion in Equation (5), we have

$$
f(x ; a, \boldsymbol{\xi})=\frac{a^{2}}{1+a} g(x, \boldsymbol{\xi}) \sum_{k=0}^{\infty} \frac{(-1)^{k} a^{k}}{k !} \frac{G(x ; \boldsymbol{\xi})^{k}}{\bar{G}(x ; \boldsymbol{\xi})^{k+3}} .
$$

By using the generalized binomial expansion, we can write

$$
\bar{G}(x ; \boldsymbol{\xi})^{-(k+3)}=\sum_{i=0}^{\infty} \frac{\Gamma(i+k+3)}{i ! \Gamma(k+3)} G(x ; \boldsymbol{\xi})^{i} .
$$

Inserting (18) in (17), the OL-G density function can be expressed as an infinite mixture of Exp-G density functions

$$
f(x ; a, \boldsymbol{\xi})=\sum_{i, k=0}^{\infty} \gamma_{i, k} h_{i+k+1}(x ; \boldsymbol{\xi})
$$

where

$$
\gamma_{i, k}=\frac{(-1)^{k} a^{2+k} \Gamma(i+k+3)}{(a+1)(i+k+1) i ! k ! \Gamma(k+3)} .
$$

The cdf of $X$ can be given by integrating (19) as

$$
F(x ; a, \boldsymbol{\xi})=\sum_{i, k=0}^{\infty} \gamma_{i, k} H_{i+k+1}(x ; \boldsymbol{\xi}) .
$$


The properties of Exp-G distributions have been studied by many authors in recent years, see Mudholkar and Srivastava (1993) and Mudholkar, Srivastava, and Freimer (1995) for exponentiated Weibull, Gupta, Gupta, and Gupta (1998) for exponentiated Pareto, Gupta and Kundu (1999) for exponentiated exponential, Nadarajah (2005) for exponentiated Gumbel, Shirke and Kakade (2006) for exponentiated log-normal and Nadarajah and Gupta (2007) for exponentiated gamma distributions.

Thus, some structural properties of the new family such as the ordinary and incomplete moments and generating function can be determined from well-established properties of the Exp-G distributions.

Note that Equations (19) and (20) are the main results of this section.

\subsection{Moments}

From now on, we assume that $Y_{i+k} \sim \operatorname{Exp}-\mathrm{G}(i+k+1)$. Many of the important features and characteristics of a distribution can be obtained using ordinary moments. A first formula for the $n$th moment of $X$ can be obtained from (19) as

$$
\mu_{n}^{\prime}=E\left(X^{n}\right)=\sum_{i, k=0}^{\infty} \gamma_{i, k} E\left(Y_{i+k}^{n}\right)
$$

Closed-form expressions for moments of several Exp-G distributions are given by Nadarajah and Kotz (2006) that can be used to obtain OL-G moments. For instance, the moments of the OLW model (for $n>-\alpha$ ) discussed in Section 2 can be derived from closed-forms moments of the exponetiated Weibull given by Nadarajah and Kotz (2006). In this case, we obtain

$$
\mu_{n}^{\prime}=\lambda^{-n} \Gamma(n / \alpha+1) \sum_{i, k=0}^{\infty}(i+k+1) \gamma_{i, k} \sum_{j=0}^{\infty} \frac{(-i-k)_{j}}{j !(j+1)^{n / \alpha+1}}
$$

A second alternative formula for $\mu_{n}^{\prime}$ can be obtained from (21) in terms of the baseline quantile function $Q_{G}(u)$. We obtain

$$
\mu_{n}^{\prime}=\sum_{i, k=0}^{\infty}(i+k+1) \gamma_{i, k} \tau_{n, i+k}
$$

where the integral depends on the baseline qf

$$
\tau_{n, j}=\int_{0}^{1} Q_{G}(u)^{n} u^{j} d u
$$

Note that the ordinary moments of several OL-G distributions can be determined directly from Equations (23) and (24). We now provide the PWMs (Probability Weighted Moments) for one distribution discussed in Section 2. Cordeiro and Nadarajah (2011) determined $\tau_{r, s}$ for some well-known distribution such as normal, beta, gamma, and Weibull distributions, which can be applied to obtain raw moments of the corresponding OL-G distributions.

For instance, in OL-N distribution discussed in Section 2, the quantities $\tau_{r, s}$ can be expressed in terms of the Lauricella functions of type A, Exton (1978) and Trott (2006) defined by

$$
\begin{aligned}
& F_{A}^{(n)}\left(a ; b_{1}, \ldots, b_{n} ; c_{1}, \ldots, c_{n} ; x_{1}, \ldots, x_{n}\right)= \\
& \sum_{m 1=0}^{\infty} \ldots \sum_{m_{n}=0}^{\infty} \frac{(a)_{m_{1}+\ldots+m_{n}}\left(b_{1}\right)_{m_{1}} \ldots\left(b_{n}\right)_{m_{n}}}{\left(c_{1}\right)_{m_{1}} \ldots\left(c_{n}\right)_{m_{n}}} \frac{x_{1}^{m_{1}} \ldots x_{n}^{m_{n}}}{m_{1} ! \ldots m_{n} !},
\end{aligned}
$$

where $(a)_{i}=a(a+1) \ldots(a+i-1)$ is the ascending factorial given by (with the convention that $\left.(a)_{0}=1\right)$. 
In fact, Cordeiro and Nadarajah (2011) determined $\tau_{r, s}$ for the standard normal distribution as

$$
\begin{gathered}
\tau_{r, s}=2^{r / 2} \pi^{-(s+1 / 2)} \sum_{\begin{array}{c}
l=0 \\
(r+s-l) \text { even }
\end{array}}^{s}\left(\begin{array}{l}
s \\
l
\end{array}\right) 2^{-l} \pi^{l} \Gamma\left(\frac{r+s-l+1}{2}\right) \times \\
F_{A}^{(s-l)}\left(\frac{r+s-l+1}{2} ; \frac{1}{2}, \ldots, \frac{1}{2} ; \frac{3}{2}, \ldots, \frac{3}{2} ;-1, \ldots,-1\right) .
\end{gathered}
$$

This equation holds when $r+s-l$ is even and it vanishes when $r+s-l$ is odd. So, any OLN moment can be expressed as an infinite weighted linear combination of Lauricella functions of type A.

Further, the central moments $\left(\mu_{r}\right)$ and cumulants $\left(\kappa_{r}\right)$ of $X$ can be determined from the ordinary moments using the recurrence equations

$$
\mu_{r}=\sum_{k=0}^{r}(-1)^{k}\left(\begin{array}{l}
r \\
k
\end{array}\right) \mu_{1}^{\prime k} \mu_{r-k}^{\prime} \quad \text { and } \quad \kappa_{r}=\mu_{r}^{\prime}-\sum_{k=1}^{r-1}\left(\begin{array}{l}
r-1 \\
k-1
\end{array}\right) \kappa_{k} \mu_{r-k}^{\prime}
$$

respectively, where $\kappa_{1}=\mu_{1}^{\prime}$. Then, $\kappa_{2}=\mu_{2}^{\prime}-\mu_{1}^{\prime 2}, \kappa_{3}=\mu_{3}^{\prime}-3 \mu_{2}^{\prime} \mu_{1}^{\prime}+2 \mu_{1}^{\prime 3}, \kappa_{4}=\mu_{4}^{\prime}-4 \mu_{3}^{\prime} \mu_{1}^{\prime}-$ $3 \mu_{2}^{\prime 2}+12 \mu_{2}^{\prime} \mu_{1}^{\prime 2}-6 \mu_{1}^{\prime 4}$, etc. The skewness $\rho_{1}=\kappa_{3} / \kappa_{2}^{3 / 2}$ and kurtosis $\rho_{2}=\kappa_{4} / \kappa_{2}^{2}$ can be obtained from the second, third and fourth cumulants.

The incomplete moments play an important role for measuring inequality. For example, the main application of the first incomplete moment refers to the Lorenz and Bonferroni curves. The $n$th incomplete moment of $X$ is calculated as

$$
m_{n}(y)=E\left(X^{n} \mid X<y\right)=\sum_{i, k=0}^{\infty}(i+k+1) \gamma_{i, k} \int_{0}^{G(y)} Q_{G}(u)^{n} u^{i+k} d u .
$$

The last integral can be evaluated for most baseline $G$ distributions.

\subsection{Generating function}

Here, we provide two formulae for the $\operatorname{mgf} M(t)=M(t ; a, \boldsymbol{\xi})=E[\exp (t X)]$ of $X$. The first one comes from (19) as

$$
M(t)=\sum_{i, k=0}^{\infty} \gamma_{i, k} M_{i+k}(t)
$$

where $M_{i+k}(t)$ is the mgf of $Y_{i+k}$. Hence, $M(t)$ can be determined from the generating function of the Exp- $G$ distribution.

A second formula for $M(t)$ can be derived from (19) as

$$
M(t)=\sum_{i, k=0}^{\infty}(i+k+1) \gamma_{i, k} \rho(t, i+k),
$$

where

$$
\rho(t, b)=\int_{-\infty}^{\infty} \exp (t x) G(x)^{b} g(x) d x=\int_{0}^{1} \exp \left\{t Q_{G}(u)\right\} u^{b} d u
$$

We can obtain the mgf of several OL-G distributions directly from Equations (26) and (27). These equations are the main results of this section. 


\section{Other measures}

\subsection{Entropy}

The entropy of a random variable $X$ with density function $f(x)$ is a measure of variation of the uncertainty. Two popular entropy measures are due to Shannon (1951) and Rényi (1961). A large value of the entropy indicates the greater uncertainty in the data. The Rényi entropy is defined by (for $\gamma>0$ and $\gamma \neq 1$ )

$$
I_{R}(\gamma)=\frac{1}{1-\gamma} \log \left(\int_{0}^{\infty} f^{\gamma}(x) d x\right) .
$$

The Shannon entropy is given by $E\{-\log [f(X)]\}$. It is a special case of the Rényi entropy when $\gamma \uparrow 1$.

Here, we derive expressions for the Rényi entropy for the OL-G distribution. Due to the fact that the parameter $\gamma$ is not in general a natural number, it is difficult to use (19) for entropy derivation. So, we use (5), the power series for the exponential and the generalized binomial expansion to obtain the Rényi entropy of $\mathrm{X}$ as

$$
I_{R}(\gamma)=\frac{1}{1-\gamma}\left\{\gamma \log \left(\frac{a^{2}}{1-a}\right)+\log \left[\sum_{i, k=0}^{\infty} \frac{(-1)^{k}(a \gamma)^{k} \Gamma(3 \gamma+i+k)}{i ! k ! \Gamma(3 \gamma+k)} K(\gamma, i, k)\right]\right\} .
$$

Here, $K(\gamma, i, k)$ denotes the integral

$$
K(\gamma, i, k)=\int_{0}^{1} g^{\gamma-1}\left[Q_{G}(u)\right] u^{i+k} d u,
$$

to be evaluated for each OL-G model. For the OL-exponential (with parameter $\lambda>0$ ), OL-Weibull (with parameters $\alpha>0$ and $\lambda>0$ ) and OL-Pareto (with parameter $\gamma>0$ ) distributions, we obtain

$$
\begin{aligned}
& K(\gamma, i, k)=\lambda^{\gamma-1} B(i+k, \gamma-1) \\
& K(\gamma, i, k)=\beta^{\gamma-1} \Gamma\left(\frac{(\alpha-1)(\gamma-1)}{\alpha}+1\right) \sum_{p=0}^{i+k}(-1)^{p}(p+\gamma)^{-\frac{(\alpha-1)(\gamma-1)}{\alpha}-1}\left(\begin{array}{c}
i+k \\
p
\end{array}\right),
\end{aligned}
$$

where $\alpha>(\gamma-1) / \gamma$, and

$$
K(\gamma, i, k)=\gamma^{\gamma-1} B\left(i+k,\left(1+\gamma^{-1}\right)(\gamma-1)\right),
$$

respectively.

\subsection{Reliability}

The measure of reliability of industrial components has many applications especially in the area of engineering. The reliability of a product (system) is the probability that the product (system) will perform its intended function for a specified time period when operating under normal (or stated) environmental conditions. The component fails at the instant that the random stress $X_{2}$ applied to it exceeds the random strength $X_{1}$, and the component will function satisfactorily whenever $X_{1}>X_{2}$. Hence, $R=P\left(X_{2}<X_{1}\right)$ is a measure of component reliability (see Kotz, Lai, and Xie (2003)). We derive the reliability $R$ when $X_{1}$ and $X_{2}$ have independent $\mathrm{OL}-\mathrm{G}\left(x, a_{1}, \boldsymbol{\xi}\right)$ and $\mathrm{OL}-\mathrm{G}\left(x, a_{2}, \boldsymbol{\xi}\right)$ distributions with the same parameter vector $\boldsymbol{\xi}$ for the baseline $G$. The reliability is defined by 


$$
R=\int_{0}^{\infty} f_{1}(x) F_{2}(x) d x .
$$

The pdf of $X_{1}$ and cdf of $X_{2}$ are obtained from Equations (19) and (20) as

$$
f_{1}(x)=\sum_{i, j=0}^{\infty} p_{i, j}\left(a_{1}\right) g(x, \boldsymbol{\xi}) G(x ; \boldsymbol{\xi})^{i+j} \quad \text { and } \quad F_{2}(x)=\sum_{k, l=0}^{\infty} q_{k, l}\left(a_{2}\right) G(x ; \boldsymbol{\xi})^{k+l+1},
$$

where

$$
p_{i, j}=\frac{(-1)^{j} a_{1}^{2+j} \Gamma(i+j+3)}{i ! j !\left(a_{1}+1\right) \Gamma(j+3)}
$$

and

$$
q_{k, l}=\frac{(-1)^{l} a_{2}^{2+l} \Gamma(k+l+3)}{k ! l !\left(a_{2}+1\right)(k+l+1) \Gamma(l+3)} .
$$

Hence,

$$
R=\sum_{i, j, k, l=0}^{\infty} p_{i, j}\left(a_{1}\right) q_{k, l}\left(a_{2}\right) \int_{0}^{\infty} g(x ; \boldsymbol{\xi}) G(x ; \boldsymbol{\xi})^{i+j+k+l+1} d x .
$$

Setting $u=G(x ; \boldsymbol{\xi})$, the reliability of the OL-G distribution reduces to

$$
R=\sum_{i, j, k, l=0}^{\infty} \frac{p_{i, j}\left(a_{1}\right) q_{k, l}\left(a_{2}\right)}{i+j+k+l+2}
$$

\subsection{Order statistics}

A branch of statistics known as order statistics plays a proeminent role in real-life applications involving data relating to life testing studies. These statistics are required in many fields, such as climatology, engineering and industry, among others. A comprehensive exposition of order statistics and associated inference is provided by David and Nagaraja (2003). Let $X_{i: n}$ denote the $i$ th order statistic. The density $f_{i: n}(x)$ of the $i$ th order statistic, for $i=1, \ldots, n$, from independent and identically distributed random variables $X_{1}, \ldots, X_{n}$ having the OL-G distribution is given by

$$
f_{i: n}(x)=M f(x) F(x)^{i-1}[1-F(x)]^{n-i},
$$

where $M=n ! /[(i-1) !(n-i) !]$. First of all, by adding and subtracting $a G(x)$ in numerator of $\frac{a+\bar{G}(x)}{(1+a) \bar{G}(x)}$, the cdf (4) can be rewritten as

$$
F(x)=1-\left\{1+\frac{a}{(1+a)}\left[\frac{G(x)}{\bar{G}(x)}\right]\right\} \exp \left\{-a \frac{G(x)}{\bar{G}(x)}\right\} .
$$

From Equations (5) and (28),

$$
\begin{aligned}
f_{i: n}(x) & =M \frac{a^{2}}{1+a} \frac{g(x)}{\bar{G}(x)} \sum_{k=0}^{i-1}(-1)^{k}\left(\begin{array}{c}
i-1 \\
k
\end{array}\right)\left\{1+\frac{a}{1+a} \frac{G(x)}{\bar{G}(x)}\right\}^{k+n-i} \\
& \times \exp \left\{-a(k+n-i+1) \frac{G(x)}{\bar{G}(x)}\right\} .
\end{aligned}
$$


The folowing equations are obtained by using the power series for the exponential function and the generalized binomial expansion:

$$
\exp \left\{-a(k+n-i+1)\left[\frac{G(x)}{\bar{G}(x)}\right]\right\}=\sum_{m=0}^{\infty} \frac{(-1)^{m} a^{m}(n+k-i+1)^{m}}{m !}\left[\frac{G(x)}{\bar{G}(x)}\right]^{m}
$$

and

$$
[1-G(x)]^{-(j+m+1)}=\sum_{p=0}^{\infty}\left(\begin{array}{c}
j+m+p \\
j+m
\end{array}\right) G(x)^{p}
$$

Based on Equations (29) and (30), the density of the order statistic $X_{i: n}$ can be expressed as a mixture of Exp-G densities.

$$
f_{i: n}(x)=\sum_{m, p=0}^{\infty} \sum_{j=0}^{k+n-i} \gamma_{j, m, p} h_{j+m+p}(x),
$$

where

$$
\gamma_{j, m, p}=\frac{M a^{j+m+2}}{m !(1+a)^{j+1}(j+m+p+1)}\left(\begin{array}{c}
j+m+p \\
j+m
\end{array}\right) \sum_{k=0}^{i-1}(-1)^{k+m}\left(\begin{array}{c}
k+n-i \\
j
\end{array}\right)\left(\begin{array}{c}
i-1 \\
k
\end{array}\right) .
$$

Clearly, the cdf of $X_{i: n}$ can be expressed as

$$
F_{i: n}(x)=\sum_{m, p=0}^{\infty} \sum_{j=0}^{k+n-i} \gamma_{j, m, p} H_{j+m+p}(x) .
$$

Hence, several mathematical quantities of the OL-G order statistics such as the ordinary, incomplete and factorial moments, mgf and mean deviations can be determined from those quantities of the Exp-G distributions. For example, from Equation (31), the moments and mgf of $X_{i: n}$ are given by

$$
E\left(X_{i: n}^{s}\right)=\sum_{m, p=0}^{\infty} \sum_{j=0}^{k+n-i} \gamma_{j, m, p} E\left(Z_{j+m+p}^{s}\right)
$$

and

$$
M_{i: n}(t)=\sum_{m, p=0}^{\infty} \sum_{j=0}^{k+n-i} \gamma_{j, m, p} E\left(\mathrm{e}^{t Z_{j+m+p}}\right),
$$

where $Z_{j+m+p} \sim \operatorname{Exp}-\mathrm{G}(j+m+p)$. Equations (31) and (32) are the main results of this section.

\section{4. $K$ upper record values}

Chandler (1952) formulated the theory of record values as a model for successive extremes in a sequence of independently and identically random variables. Record values are found in many real life applications involving data related to economics, sports, weather and life testing problems. The statistical study of record values has now spread in various directions. Dziubdziela and Kopocinski (1976) proposed the model of $k$ upper record values by observing successive $k$ largest values in a sequence, where $k$ is a positive integer.

Let $X_{n}^{(k)}$ denote the $k$ th upper record value. The pdf $f_{X_{n}^{(k)}}(x)$ of the $k$ th upper record value, for $k=1, \ldots, n$, from independent and identically distributed random variables $X_{1}, \ldots, X_{n}$ from the OL-G distribution is given by

$$
f_{X_{n}^{(k)}}(x)=\frac{k^{n}}{(n-1) !}\{-\log [1-F(x)]\}^{n-1}[1-F(x)]^{k-1} f(x) .
$$


By expanding the logarithm function in power series and using the binomial expansion, we have

$$
f_{X_{n}^{(k)}}(x)=\frac{k^{n}}{(n-1) !}\left\{\sum_{p=0}^{\infty} a_{p} F(x)^{p+1}\right\}^{n-1}\left\{\sum_{j=0}^{k-1}(-1)^{j}\left(\begin{array}{c}
k-1 \\
j
\end{array}\right) F(x)^{j}\right\} f(x),
$$

where $a_{p}=1 /(p+1)$.

Here, we use an equation by Gradshteyn and Ryzhik (2007) (Section 0.314) for a power series raised to a positive integer $n$

$$
\left(\sum_{i=0}^{\infty} a_{i} u^{i}\right)^{n}=\sum_{i=0}^{\infty} c_{n, i} u^{i}
$$

where the coefficients $c_{n, i}$ (for $i=1,2, \ldots$ ) are determined from the recurrence equation

$$
c_{n, i}=\left(i a_{0}\right)^{-1} \sum_{m=1}^{i}[m(n+1)-i] a_{m} c_{n, i-m},
$$

where $c_{n, 0}=a_{0}^{n}$.

Based on Equations (34) and (35), the pdf (33) can be expressed as

$$
f_{X_{n}^{(k)}}(x)=\frac{k^{n}}{(n-1) !} \sum_{p=0}^{\infty} \sum_{j=0}^{k-1}(-1)^{j} c_{n-1, p}\left(\begin{array}{c}
k-1 \\
j
\end{array}\right) F(x)^{j+n+p-1} f(x),
$$

where $c_{n-1, p}$ can be obtained from the quantities $a_{0}, \ldots, a_{p}$ as in Equation (35).

From Equations (5) and (28), and following similar algebra of Section 4.3, we obtain

$$
f_{X_{n}^{(k)}}(x)=\sum_{r=0}^{q} \sum_{s, t=0}^{\infty} \phi_{r, s, t} h_{r+s+t+1}(x)
$$

where

$$
\begin{aligned}
\phi_{r, s, t} & =\left(\begin{array}{l}
n \\
k
\end{array}\right)\left(\begin{array}{c}
r+s+t+2 \\
r+s+2
\end{array}\right) \frac{k^{n} a^{r+s+2}}{(r+s+t+1)(n+1) !(1+a)^{r+1}} \\
& \times \sum_{p=0}^{\infty} \sum_{j=0}^{k-1} \sum_{q=0}^{j+n+p-1}\left(\begin{array}{c}
q \\
r
\end{array}\right)\left(\begin{array}{c}
k-1 \\
j
\end{array}\right)(-1)^{j+q}(q+1)^{s} c_{n-1, p} .
\end{aligned}
$$

Equation (36) is the main result of this section. It reveals that the pdf of the OL-G $k$ upper record values is a triple linear combination of Exp-G densities.

\section{Estimation}

We determine the maximum likelihood estimates (MLEs) of the parameters of the new family from complete samples only. Let $x_{1}, \ldots, x_{n}$ be observed values from the OL-G distribution with parameters $a$ and $\boldsymbol{\xi}$. Let $\boldsymbol{\Theta}=(a, \boldsymbol{\xi})^{\top}$ be the $p \times 1$ parameter vector. The total loglikelihood function for $\Theta$ is given by

$$
\ell(\Theta)=2 n \log (a)-n \log (1+a)+\sum_{i=1}^{n} \log \left[g\left(x_{i} ; \boldsymbol{\xi}\right)\right]-3 \sum_{i=1}^{n} \log \left[\bar{G}\left(x_{i} ; \boldsymbol{\xi}\right)\right]-a \sum_{i=1}^{n} V\left(x_{i} ; \boldsymbol{\xi}\right),
$$

where $V(x ; \boldsymbol{\xi})=G(x ; \boldsymbol{\xi}) / \bar{G}(x ; \boldsymbol{\xi})$. We assume that the folllowing standard regularity conditions for the log-likelhood $\ell(\boldsymbol{\Theta})$ hold: i) The support of $X$ associated to the distribution does 
not depend on unknown parameters; ii) The parameter space of $X$, say $\boldsymbol{\Psi}$ is open and $\ell(\boldsymbol{\Theta})$ has a global maximum in $\mathbf{\Psi}$; iii) For almost all $x$, the fourth-order log-likelihood derivatives with respect to the model parameters exist and are continuous in an open subset of $\boldsymbol{\Psi}$ that contains the true parameter; iv) The expected information matrix is positive definite and finite; v) The absolute values of the third-order log-likelihood derivatives with respect to the parameters are bounded by expected finite functions of $X$.

The components of the score function $U(\boldsymbol{\Theta})=\left(U_{a}, U_{\boldsymbol{\xi}}\right)^{\top}$ are

$$
U_{a}=\frac{2 n}{a}-\frac{n}{1+a}-\sum_{i=1}^{n} V\left(x_{i} ; \boldsymbol{\xi}\right)
$$

and

$$
U_{\boldsymbol{\xi}_{k}}=-a \sum_{i=1}^{n} \partial V\left(x_{i} ; \boldsymbol{\xi}\right) / \partial \boldsymbol{\xi}_{k}+\sum_{i=1}^{n} \frac{\partial g\left(x_{i} ; \boldsymbol{\xi}\right) / \partial \boldsymbol{\xi}_{k}}{g\left(x_{i} ; \boldsymbol{\xi}\right)}-3 \sum_{i=1}^{n} \frac{\partial \bar{G}\left(x_{i} ; \boldsymbol{\xi}\right) / \partial \boldsymbol{\xi}_{k}}{\bar{G}\left(x_{i} ; \boldsymbol{\xi}\right)}
$$

Setting $U_{a}$ and $U_{\boldsymbol{\xi}}$ equal to zero and solving the equations simultaneously yields the MLE $\widehat{\boldsymbol{\Theta}}=(\widehat{a}, \widehat{\boldsymbol{\xi}})^{\top}$ of $\boldsymbol{\Theta}=(a, \boldsymbol{\xi})^{\top}$. These equations cannot be solved analytically and statistical software can be used to solve them numerically using iterative methods such as the NewtonRaphson type algorithms.

For interval estimation on the model parameters, we obtain the $(p+1) \times(p+1)$ observed information matrix $J(\boldsymbol{\Theta})=\left\{U_{r s}\right\}$ (for $r, s=a, \boldsymbol{\xi}_{\boldsymbol{k}}$ ), whose elements are

$$
U_{a a}=-\frac{2 n}{a^{2}}+\frac{n}{(1+a)^{2}}, \quad U_{a \boldsymbol{\xi}_{k}}=-\sum_{i=1}^{n} \partial V\left(x_{i} ; \boldsymbol{\xi}\right) / \partial \boldsymbol{\xi}_{k}
$$

and

$$
\begin{aligned}
U_{\xi_{k} \xi_{l}} & =-a \sum_{i=1}^{n} \partial^{2} V\left(x_{i} ; \boldsymbol{\xi}\right) / \partial \boldsymbol{\xi}_{k} \partial \boldsymbol{\xi}_{l}-\sum_{i=1}^{n} \frac{\left[\partial g\left(x_{i} ; \boldsymbol{\xi}\right) / \partial \boldsymbol{\xi}_{k}\right]\left[\partial g\left(x_{i} ; \boldsymbol{\xi}\right) / \partial \boldsymbol{\xi}_{l}\right]}{g\left(x_{i} ; \boldsymbol{\xi}\right)^{2}}+\sum_{i=1}^{n} \frac{\partial^{2} g\left(x_{i} ; \boldsymbol{\xi}\right) / \partial \boldsymbol{\xi}_{k} \partial \boldsymbol{\xi}_{l}}{g\left(x_{i} ; \boldsymbol{\xi}\right)} \\
& +3 \sum_{i=1}^{n} \frac{\left[\partial \bar{G}\left(x_{i} ; \boldsymbol{\xi}\right) / \partial \boldsymbol{\xi}_{k}\right]\left[\partial \bar{G}\left(x_{i} ; \boldsymbol{\xi}\right) / \partial \boldsymbol{\xi}_{l}\right]}{\bar{G}\left(x_{i} ; \boldsymbol{\xi}\right)^{2}}-3 \sum_{i=1}^{n} \frac{\partial^{2} \bar{G}\left(x_{i} ; \boldsymbol{\xi}\right) / \partial \boldsymbol{\xi}_{k} \partial \boldsymbol{\xi}_{l}}{\bar{G}\left(x_{i} ; \boldsymbol{\xi}\right)}
\end{aligned}
$$

\section{Empirical and numerical illustration}

\subsection{Some numerical values and simulation}

The formulae derived in this paper can be easily handled in most symbolic computation software platforms such as MAPLE, MATLAB and MATHEMATICA. These platforms have currently the ability to deal with complex expressions. Table 2 provides some numerical values for the ordinary moments $\mu_{i}$ (with $i=1,2,3,4$.) and quantiles $Q(u)$ of the $\operatorname{OLW}(a, \alpha, \lambda)$ distribution calculated from MATHEMATICA. These moments were established using numerical integration. The quantile values were obtained by using Equation (14). The values of the Lambert $\mathrm{W}$ function is avaliable in routine ProductLog $[\cdot, \cdot]$. 
Table 2: The values of the first four moments and some quantiles of the $\operatorname{OLW}(a, \alpha, \lambda)$ distribution for $\alpha=1.0, \lambda=4.0$ and different values of $a$

\begin{tabular}{llllll}
\hline OLW for $a:$ & 0.01 & 0.2 & 1.0 & 1.5 & 2.0 \\
\hline$\mu_{1}$ & 1.257 & 0.519 & 0.199 & 0.144 & 0.113 \\
$\mu_{2}$ & 1.620 & 0.303 & 0.053 & 0.030 & 0.019 \\
$\mu_{3}$ & 2.13 & 0.189 & 0.017 & 0.007 & 0.004 \\
$\mu_{4}$ & 2.850 & 0.125 & 0.005 & 0.002 & $<0.001$ \\
$Q(0.1)$ & 0.993 & 0.266 & 0.045 & 0.027 & 0.018 \\
$Q(0.3)$ & 1.174 & 0.433 & 0.122 & 0.079 & 0.057 \\
$Q(0.7)$ & 1.374 & 0.627 & 0.262 & 0.191 & 0.149 \\
$Q(0.9)$ & 1.490 & 0.743 & 0.363 & 0.280 & 0.228 \\
\hline OLW for $a:$ & 0.3 & 0.5 & 0.7 & 0.9 & 1.1 \\
\hline$\mu_{1}$ & 0.427 & 0.320 & 0.257 & 0.215 & 0.185 \\
$\mu_{2}$ & 0.212 & 0.126 & 0.085 & 0.062 & 0.047 \\
$\mu_{3}$ & 0.114 & 0.055 & 0.032 & 0.020 & 0.014 \\
$\mu_{4}$ & 0.066 & 0.026 & 0.013 & 0.007 & 0.004 \\
$Q(0.2)$ & 0.276 & 0.178 & 0.127 & 0.096 & 0.076 \\
$Q(0.5)$ & 0.439 & 0.323 & 0.255 & 0.208 & 0.175 \\
$Q(0.6)$ & 0.483 & 0.366 & 0.294 & 0.244 & 0.209 \\
$Q(0.8)$ & 0.579 & 0.457 & 0.381 & 0.327 & 0.286 \\
\hline
\end{tabular}

We assess the performance of the MLEs of the OLW distribution with respect to sample size $n$. The assessment was based on a simulation study:

1. generate ten thousand samples of size $n$ from (4)-(5). The inversion method was used to generate samples.

2. compute the MLEs for the ten thousand samples, say $(\widehat{a}, \widehat{\alpha}, \widehat{\lambda})$ for $i=1,2, \ldots, 10000$.

3. compute the standard errors of the MLEs for the ten thousand samples, say $\left(s_{\widehat{a}}, s_{\widehat{\alpha}}, s_{\widehat{\lambda}}\right)$ for $i=1,2, \ldots, 10000$. The standard errors were computed by inverting the observed information matrices.

4. compute the biases and mean squared errors given by

$$
\begin{aligned}
\operatorname{bias}_{\epsilon}(n) & =\frac{1}{10000} \sum_{i=1}^{10000}\left(\widehat{\epsilon}_{i}-\epsilon\right), \\
\operatorname{MSE}_{\epsilon}(n) & =\frac{1}{10000} \sum_{i=1}^{10000}\left(\widehat{\epsilon}_{i}-\epsilon\right)^{2},
\end{aligned}
$$

for $\epsilon=a, \alpha, \lambda$.

We repeated these steps for $n=30,31, \ldots, 100$ with $a=1.2, \alpha=2.5$ and $\lambda=4.0$, so computing $\operatorname{bias}_{\epsilon}(n)$ and $\operatorname{MSE}_{\epsilon}(n)$.

Figure 5 shows how the four biases vary with respect to $n$. The biases for each parameter either decrease to zero as $n \rightarrow \infty$. The reported observations are for only one choice for $(a, \alpha, \lambda)$, namely that $(a, \alpha, \lambda)=(1.2,2.5,4.0)$. But the results were similar for a wide range of other choices for $(a, \alpha, \lambda)$. Figure 6 shows how the four mean squared errors vary with 

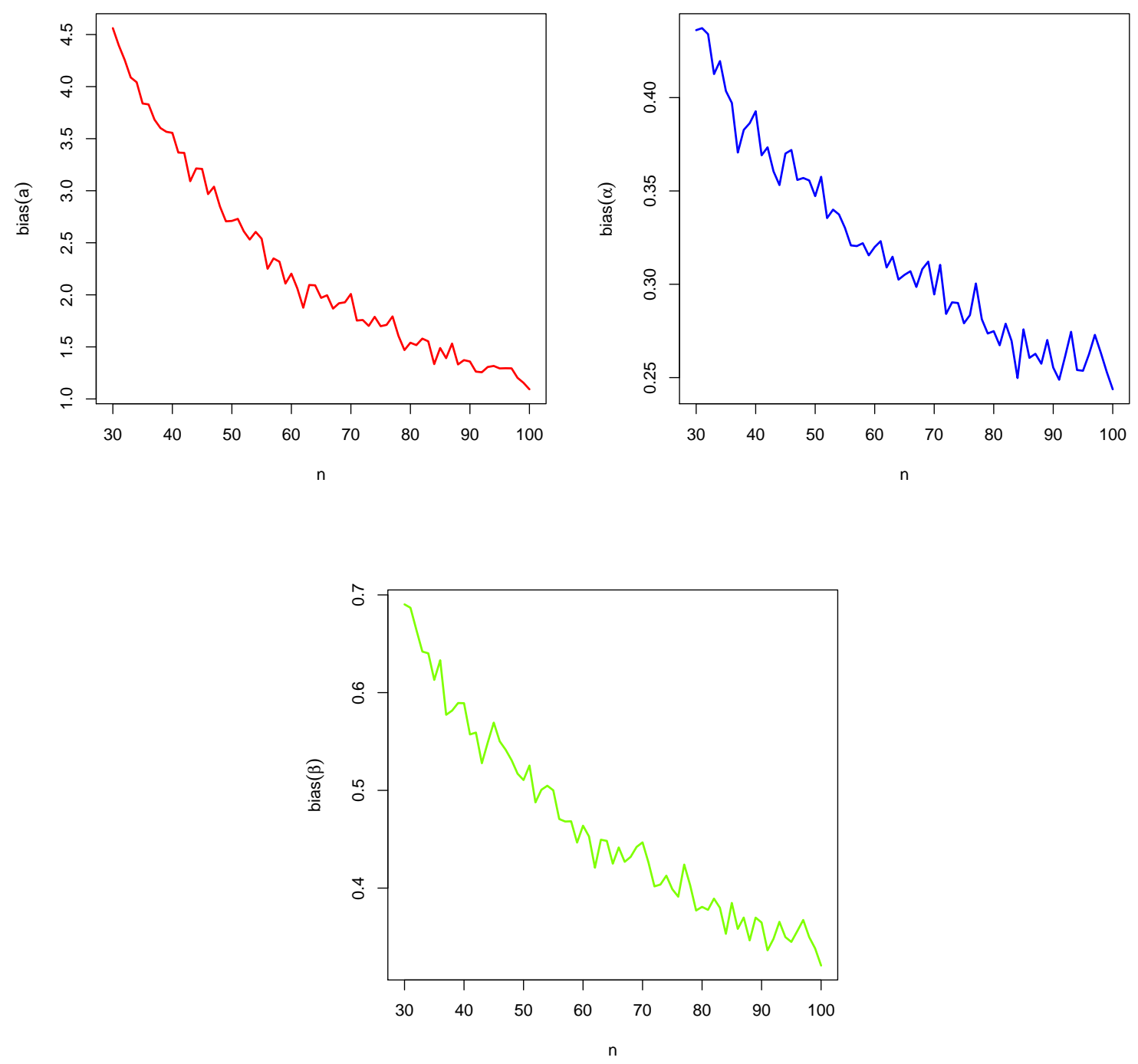

Figure 5: Biases of $\widehat{a}, \widehat{\alpha}$ and $\widehat{\lambda}$ versus $n$.

respect to $n$. The mean squared errors for each parameter decrease to zero as $n \rightarrow \infty$. In particular, i) the biases for each parameter either decreased to zero and appeared reasonably small at $n=100$; ii) the mean squared errors for each parameter decreased to zero and appeared reasonably small at $n=100$.

\subsection{Application}

We illustrate the flexibility of the OLW distribution by means of a real data set. Similar investigations could be performed for other OL-G distributions. We choose the Weibull as baseline because of its popularity. The computations are performed using the software $\mathrm{R}$ version 3.0.3 (package bbmle). The maximization follows the BFGS method with analytical derivatives. The algorithm used to estimate the model parameters converged for all current models.

The data set consists of 63 observations of the strengths of $1.5 \mathrm{~cm}$ glass fibres, originally obtained by workers at the UK National Physical Laboratory. Unfortunately, the units of measurement are not given in the paper. These data have also been analyzed by Bourguignon et al. (2014). For these data, we compare the fits of the OLW distribution defined by (6), 

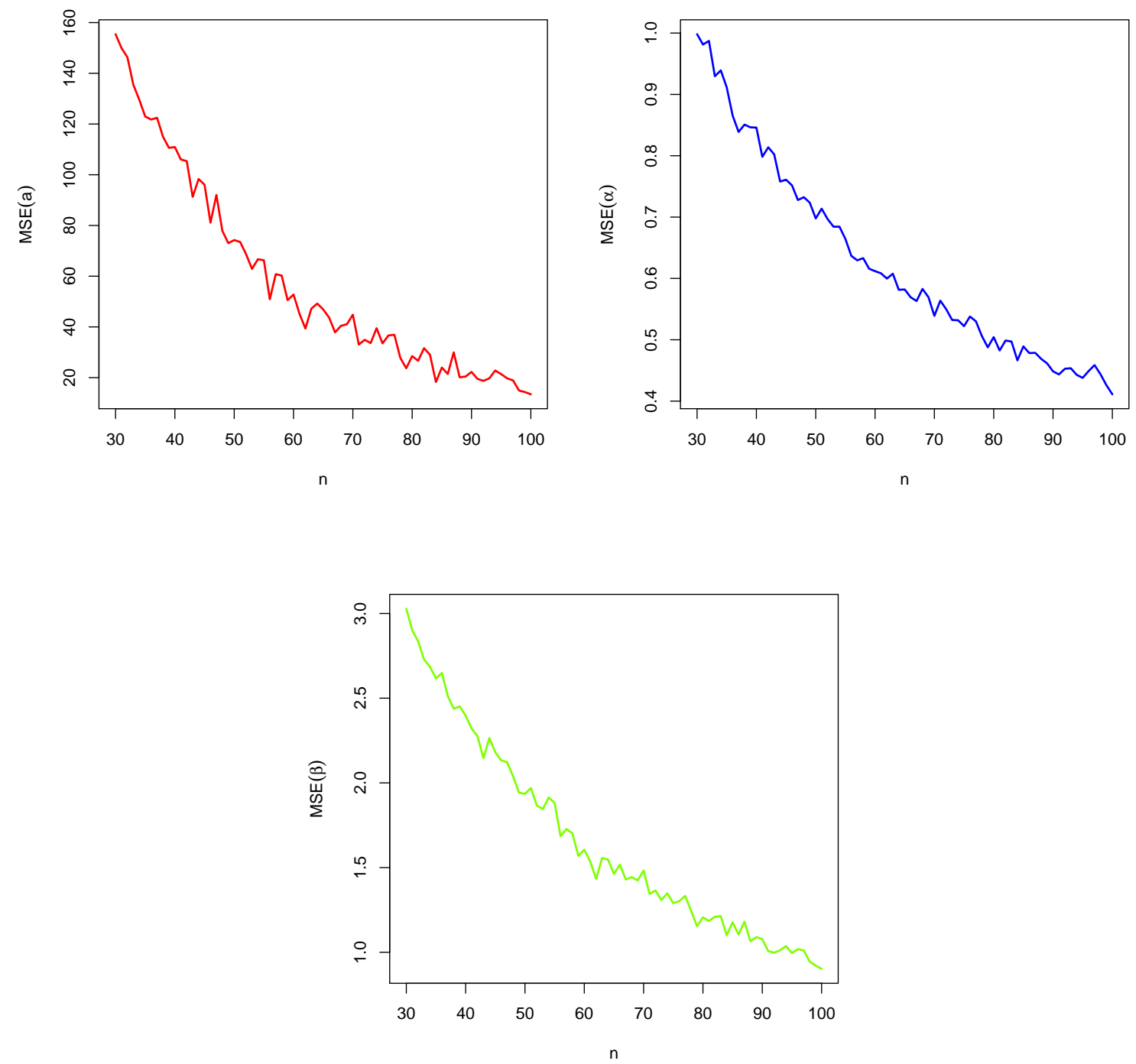

Figure 6: Mean squared errors of $\widehat{a}, \widehat{\alpha}$ and $\widehat{\lambda}$ versus $n$.

its special Weibull model (W) and of the following distributions: the exponentiated Weibull (EW) with pdf given by

$$
f_{\mathrm{EW}}(x)=a \lambda \alpha^{\lambda} x^{\lambda-1}\left(1-\mathrm{e}^{-(\alpha x)^{\lambda}}\right)^{a-1} \mathrm{e}^{-(\alpha x)^{\lambda}},
$$

the beta Weibull (BW) with pdf given by

$$
f_{\mathrm{BW}}(x)=\frac{1}{B(a, b)} \alpha^{\lambda} x^{\lambda-1} \mathrm{e}^{-(\alpha x)^{\lambda b}}\left(1-\mathrm{e}^{-(\alpha x)^{\lambda}}\right)^{a-1}
$$

and the Kumaraswamy Weibull $(\mathrm{KwW})$ with pdf given by

$$
f_{\mathrm{KwW}}(x)=a b \lambda \alpha^{\lambda} x^{\lambda-1} \mathrm{e}^{-(\alpha x)^{\lambda}}\left[1-\mathrm{e}^{-(\alpha x)^{\lambda}}\right]^{a-1}\left\{1-\left[1-\mathrm{e}^{-(\alpha x)^{\lambda}}\right]^{a}\right\}^{b-1} .
$$

All parameters of these distribution are positive numbers. In Table 3, the MLEs and their standard errors (SEs) (in parentheses) of the parameters from the five fitted models and the values of the Akaike Information Criterion (AIC), Cramér-von Mises ( $\mathrm{W}^{*}$ ) and AndersonDarling $\left(\mathrm{A}^{*}\right)$ goodness-of-fit statistics are presented. According to the lowest values of the AIC, $\mathrm{W}^{*}$ and $\mathrm{A}^{*}$ statistics, the OLW model could be chosen as the best model among the five fitted models. 
Table 3: MLEs (SEs in parentheses) for some fitted models to the strengths data and the $\mathrm{AIC}, \mathrm{W}^{*}$ and $\mathrm{A}^{*}$ values

\begin{tabular}{rrrrrccc}
\hline Model & $a$ & $b$ & $\alpha$ & $\lambda$ & $A I C$ & $W^{*}$ & $A^{*}$ \\
\hline \multirow{2}{*}{$\mathrm{W}$} & - & - & 5.781 & 1.628 & 34.414 & 0.237 & 1.304 \\
& - & - & $(0.576)$ & $(0.037)$ & & & \\
EW & 0.671 & - & 7.285 & 1.718 & 35.351 & 0.636 & 3.484 \\
& $(0.249)$ & - & $(1.707)$ & $(0.086)$ & & & \\
BW & 0.620 & 10.249 & 7.759 & 2.382 & 37.179 & 0.196 & 1.089 \\
& $(0.248)$ & $(95.117)$ & $(2.023)$ & $(2.897)$ & & & \\
KwW & 0.606 & 0.214 & 6.908 & 1.337 & 35.252 & 0.161 & 0.908 \\
& $(0.162)$ & $(0.029)$ & $(0.004)$ & $(0.003)$ & & & \\
OLW & 0.049 & - & 1.102 & 0.492 & 34.387 & 0.153 & 0.870 \\
& $(0.087)$ & - & $(0.527)$ & $(0.494)$ & & & \\
\hline
\end{tabular}

The plots of the fitted OLW pdf and of the two better fitted pdfs are displayed in Figure 7. The QQ plots for the fitted models are displayed in Figure 8. These plots indicate that the OLW distribution provides a better fit to these data compared to the other models. Finally, the proposed distribution can be considered a very competitive model to the EW distribution.

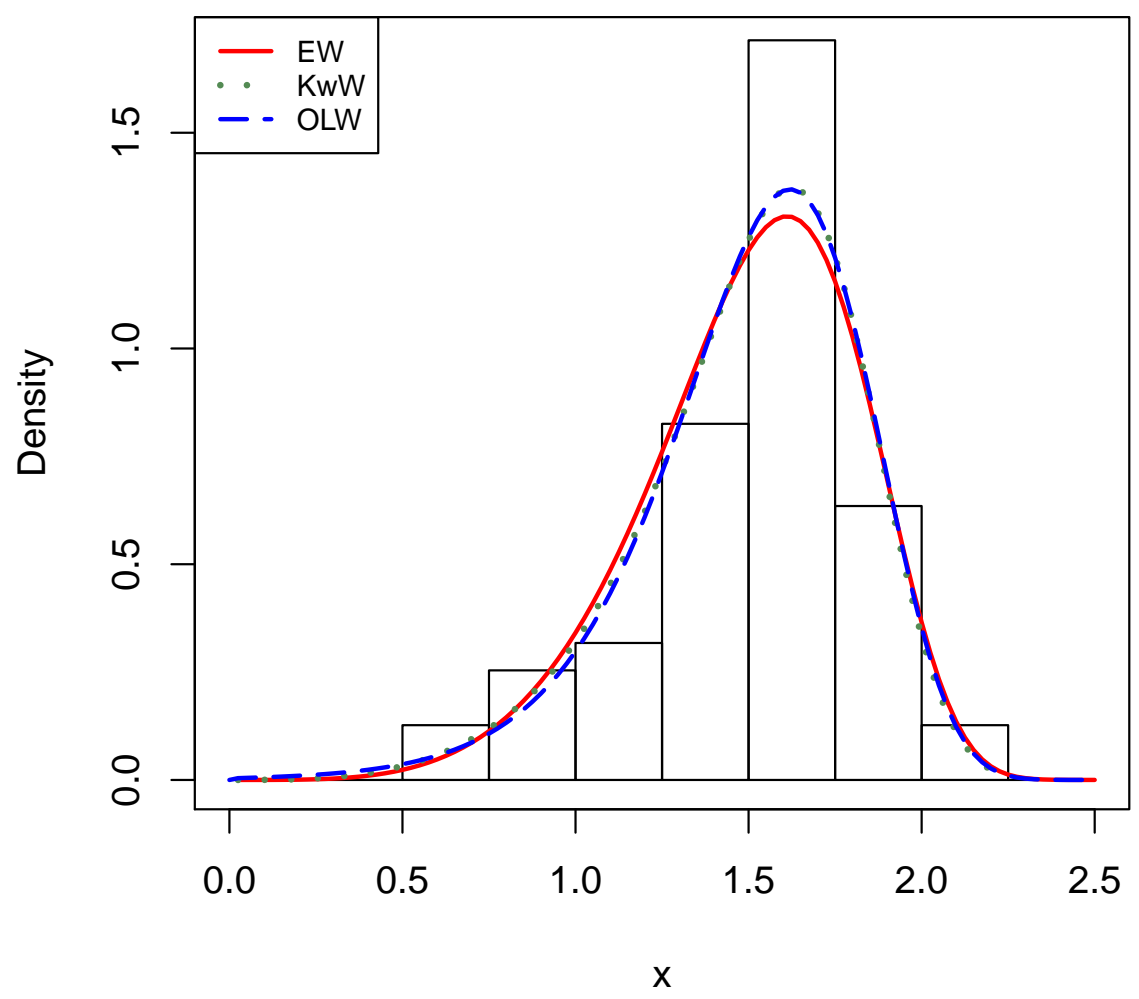

Figure 7: Fitted densities for the strengths data 
W

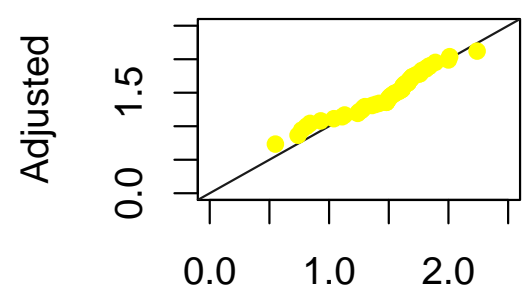

Observed

BW

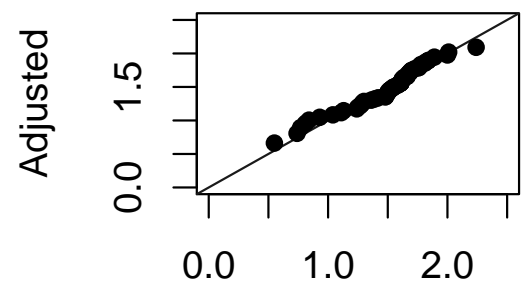

Observed
EW

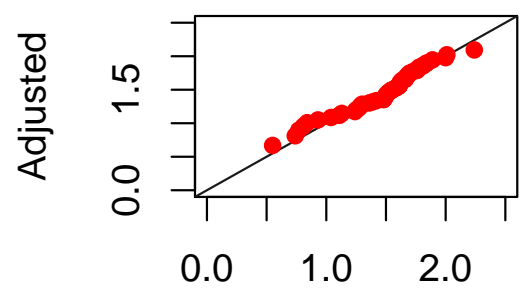

Observed

OLW

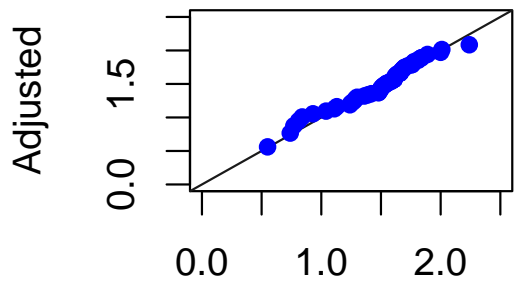

Observed

Figure 8: QQ plots for the strengths data

\section{Conclusion remarks}

In this paper we propose and study a new class of distributions called the odd Lindley$G$ family (OL-G). This family can extend several widely known models such as the Weibull, Kumaraswamy, half-logistic and Burr XII distributions in order to provide more flexibility. We investigate several of its structural properties such as an expansion for the density function and explicit expressions for the quantile function, ordinary and incomplete moments, generating function, Rényi entropy, reliability, order statistics and $k$ upper record values. We estimate the parameters using maximum likelihood and determine the observed information matrix. We also discuss inference on the parameters based on Cramér-von Mises and Anderson-Darling statistics. An example to real data proves empirically the importance and potentiality of the proposed family.

\section{References}

Alexander C, Cordeiro GM, Ortega EMM, Sarabia JM (2012). "Generalized Beta-generated Distributions." Computational Statistics and Data Analysis, 56, 1880-1897.

Alizadeh M, Emadi M, Doostparast M, Cordeiro GM, Ortega EMM, Pescim RR (2015). "A New Family of Distributions: The Kumaraswamy Odd Log-logistic, Properties and Applications." Hacet. J. Math Stat, forthcoming.

Alzaatreh A, Lee C, Famoye F (2013). "A New Method for Generating Families of Distributions." Metron, 71, 63-79. 
Alzaghal A, Felix F, Carl L (2013). "Exponentiated T-X Family of Distributions with Some Applications." International Journal of Statistics and Probability, 2, 31-49.

Asgharzedah A, Bakouch HS, Esmaeli H (2013). "Pareto Poisson-Lindley Distribution with Applications." Journal of Applied Statistics, 40, 1717-1734.

Bourguignon M, Silva RB, Cordeiro GM (2014). "The Weibull-G Family of Probability Distributions." Journal of Data Science, 12, 53-68.

Chandler KN (1952). "The Distribution and Frequency of Record Values." J. Roy. Statist. Soc., Ser B, 14, 220-228.

Cooray K (2006). "Generalization of the Weibull Distribution: The Odd Weibull Family." Statistical Modelling, 6, 265-277.

Cordeiro GM, Alizadeh M, Ortega EMM (2014). "The Exponentiated Half-logistic Family of Distributions: Properties and Applications." Journal of Probability and Statistics, 2014, 21 pages.

Cordeiro GM, de Castro M (2011). "A New Family of Generalized Distributions." Journal of Statistical Computation and Simulation, 81, 883-893.

Cordeiro GM, Nadarajah S (2011). "Closed-form Expressions for Moments of a Class of Beta Generalized Distributions." Brazilian Journal Probability and Statistics, 52, 14-33.

David HA, Nagaraja HN (2003). Order Statistics. John Wiley \& Sons, New Jersey.

Dziubdziela W, Kopocinski B (1976). "Limiting Properties of the kâĹŠth Record Value." Appl. Math., 15, 187-190.

Eugene N, Lee C, Famoye F (2002). "Beta-normal Distribution and Its Applications." Communications in Statistics Theory and Methods, 31, 497-512.

Exton H (1978). Handbook of Hypergeometric Integrals: Theory, Applications, Tables, Computer Programs. New York: Ellis Horwood, New York.

Ghitany ME, Atieh B, Nadarajah S (2008). "Lindley Distribution and Its Applications." Mathematics and Computers in Simulation, 78, 493-506.

Gradshteyn IS, Ryzhik IM (2007). Table of Integrals, Series, and Products. Academic Press, New York.

Gupta P, Singh B (2012). "Parameter Estimation of Lindley Distribution with Hybrid Censored Data." International Journal of System Assurance Engineering and Management, 4, $378-3751$.

Gupta RC, Gupta RD, Gupta PL (1998). "Modeling Failure Time Data by Lehman Alternatives." Communications in Statistics Theory and Methods, 27, 887-904.

Gupta RD, Kundu D (1999). "Generalized Exponential Distributions." Australian and New Zealand Journal of Statistics, 41, 173-188.

Johnson NL, Kotz S, Balakrishnan N (1994). Continuous Univariate Distributions. John Wiley, New York.

Johnson NL, Kotz S, Balakrishnan N (1995). Continuous Univariate Distributions. John Wiley and Sons, New York.

Kotz S, Lai CD, Xie M (2003). "On the Effect of Redundancy for Systems with Dependent Components." IIE Trans, 35, 1103-1110. 
Lindley DV (1958). "Fiducial Distributions and Bayes' Theorem." Journal of the Royal Statistical Society. Series B. (Methodological), 20, 102-107.

Marshall AN, Olkin I (1997). "A New Method for Adding a Parameter to a Family of Distributions with Applications to the Exponential and Weibull Families." Biometrika, 84, $641-552$.

Mazucheli J, Achcar JA (2011). "The Lindley Distribution Applied to Competing Risks Lifetime Data." Computer Methods and Programs in Biomedicine, 104, 188-192.

Mudholkar GS, Srivastava DK (1993). "Exponentiated Weibull Family for Analyzing Bathtub Failure-rate Data." IEEE Transactions on Reliability, 42, 299-302.

Mudholkar GS, Srivastava DK, Freimer M (1995). "The Exponentiated Weibull Family: A Reanalysis of the Bus-motor-failure Data." Technometrics, 37, 436-445.

Nadarajah Sand Bakouch HS, Tahmasbi R (2011). "A Generalized Lindley Distribution." Sankhya B, 73, 331-359.

Nadarajah S (2005). "The Exponentiated Gumbel Distribution with Climate Application." Environmetrics, 17, 13-23.

Nadarajah S, Gupta AK (2007). "The Exponentiated Gamma Distribution with Application to Drought Data." Calcutta Statistical Association Bulletin, 59, 29-54.

Nadarajah S, Kotz S (2006). "The Exponentiated-type Distributions." Acta Applicandae Mathematicae, 92, 97-111.

Rényi A (1961). On Measures of Entropy and Information. In: Proceedings of the 4th Berkeley Symposium on Mathematical, Statistics and Probability. University of California Press, Berkeley.

Sankaran M (1970). "The Discrete Poisson-Lindley Distribution.” Biometrics, 26, 145-149.

Shannon CE (1951). "Prediction and Entropy of Printed English." The Bell System Technical Journal, 30, 50-64.

Shirke DT, Kakade CS (2006). "On Exponentiated Lognormal Distribution." International Journal of Agricultural and Statistical Sciences, 2, 319-326.

Tahir MH, Cordeiro GM, Alzaatreh A, Mansoor M, Zubair M (2016a). "The Logistic-X Family of Distributions and Its Applications." Commun. Stat. Theory Methods, (forthcoming).

Tahir MH, Zubair M, Mansoor M, Cordeiro GM, Alizadeh M, Hamedani GG (2016b). "A New Weibull-G Family of Distributions." Hacet. J. Math. Stat., (forthcoming).

Trott M (2006). The Mathematica Guidebook for Symbolics. Springer, New York.

Warahena-Liyanage G, Pararai M (2014). "A Generalized Power Lindley Distribution with Applications." Asian Journal of Mathematics and Applications, pp. 1-23.

Zakerzadeh Y, Dolati A (2009). "Generalized Lindley Distribution." Journal of Mathematical Extension, 3, 13-25.

Zimmer WJ, Keats JB, Wang FK (1998). "The Burr XII Distribution in Reliability Analysis." J. Qual. Technol, 30, 386-394.

Zografos K, Balakrishnan N (2009). "On Families of Beta-and Generalized Gamma-generated Distribution and Associate Inference." Statistical Methodology, 6, 344-362. 


\section{Affiliation:}

Frank Gomes-Silva

Department of Statistics and Informatics

Federal Rural University of Pernambuco

52171-900, Recife, PE, Brazil

E-mail: franksinatrags@gmail.com

Ana Percontini

Department of Exact Sciences

State University of Feira de Santana

44036-900, Feira de Santana, BA, Brazil

E-mail: anappaixao@gmail.com

Edleide de Brito

Department of Statistics

Federal University of Bahia

40110-909, Salvador, BA, Brazil

E-mail: edbnet@gmail.com

Manoel W. Ramos

Department of Mathematics

Federal Institute of Paraíba

58015-020, João Pessoa, PB, Brazil

E-mail: wallace.ifpb@gmail.com

Ronaldo Venâncio

Recife Military School

52070-080, Recife, PE, Brazil

E-mail: ronaldovenanciorvs@gmail.com

Gauss M. Cordeiro

Department of Statistics

Federal University of Pernambuco

50740-540, Recife, PE, Brazil

E-mail: gausscordeiro@gmail.com

\section{Austrian Journal of Statistics}

published by the Austrian Society of Statistics

Volume 46

February 2017 http://www .ajs.or.at/

http://www.osg.or.at/

Submitted: 2016-01-16

Accepted: 2016-09-01 